\title{
Los ciclos circadianos y su influencia en la ocurrencia de accidentes en las organizaciones de Estado de México
}

Circadian Cycles and Their Influence on the Occurrence of Accidents in Organizations from the East of the Estado de México

\section{Ciclos circadianos e sua influência na ocorrência de acidentes em organizações no Estado do México}

\section{Lizette Alvarado Tarango}

Tecnológico Nacional de México, Instituto Tecnológico de Ciudad Juárez, México

lalvarado@itcj.edu.mx https://orcid.org/0000-0001-7934-8330

Juan Pedro Benítez Guadarrama Universidad Autónoma del Estado de México, México jpbenitezg@uaemex.mx https://orcid.org/0000-0002-2826-6359

Alfonso Aldape Almillo

Tecnológico Nacional de México, Instituto Tecnológico de Ciudad Juárez, México aaldape@itcj.edu.mx https://orcid.org/0000-0001-8921-9479

Francisco Zorrilla Briones

Tecnológico Nacional de México, Instituto Tecnológico de Ciudad Juárez, México

fzorrilla@itcj.edu.mx https://orcid.org/0000-0003-0553-9841

Manuel Alonso Rodríguez Morachis

Tecnológico Nacional de México, Instituto Tecnológico de Ciudad Juárez, México mmorachis@itcj.edu.mx https://orcid.org/0000-0003-1581-7737 


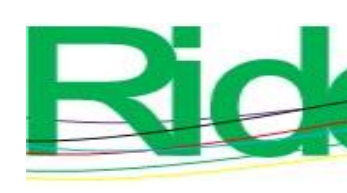

Revista Iberoamericana para la Investigación y el Desarrollo Educativo ISSN $2007-7467$

\title{
Resumen
}

Las organizaciones industriales o de transformación aspiran a contratar trabajadores para realizar actividades en los procesos de producción bajo condiciones óptimas. Sin embargo, los diferentes contextos pueden cambiar las condiciones y afectar su estado de biorritmo. Al alterarse estos factores se propicia la ocurrencia de un accidente de trabajo. El objetivo del presente trabajo es investigar la existencia de una relación entre los ciclos circadianos con las incidencias en los accidentes de trabajo en los sistemas de producción de las entidades industriales o de transformación. En este estudio longitudinal, se utilizaron las bases de datos proporcionadas por la subdelegación del Instituto Mexicano del Seguro Social (IMSS) de la zona oriente del Estado de México de los años 2013, 2014 y 2015. En el procesamiento y análisis de datos se utilizó estadística descriptiva, a fin de conocer el comportamiento de las variables en la muestra. Los resultados sugieren que los ciclos circadianos conformados por el estado físico, emocional e intelectual influyen significativamente en la ocurrencia de los accidentes de trabajo al interior de la empresa. Lo anterior permite inferir que conocer el estado biorrítmico es relevante porque, principalmente, permite ubicar y reubicar a trabajadores en periodos expuestos a riesgos y, en segunda instancia, evitar el aumento en el seguro de prima de riesgo de trabajo.

Palabras clave: accidente de trabajo, biorritmos, ciclos circadianos, riesgos laborales.

\begin{abstract}
Industrial or transformation organizations aspire to hire workers to carry out activities in the production processes under optimal conditions. However, different contexts can change conditions and affect their biorhythmic status. When these factors are altered, the occurrence of an accident at work occurs. The aim of this work is to investigate the existence of a relationship between circadian cycles with the incidence of accidents at work in the production systems of industrial or transformation entities. In this longitudinal study, the databases provided by the sub-delegation of the Instituto Mexicano del Seguro Social (IMSS) of the eastern part of the Estado de México for the years 2013, 2014 and 2015 were used.. In the data analysis, descriptive statistics of variables were applied, in order to know the behavior of the variables in the sample. The results suggest that the circadian cycles made up of the physical, emotional and intellectual state significantly influence the occurrence of workplace accidents within the company. The foregoing allows us to infer that knowing the
\end{abstract}




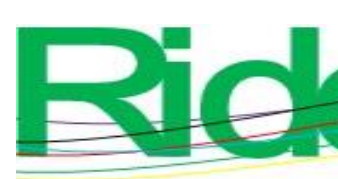

Revista Iberoamericana para la
Investigación y el Desarrollo Educativo
ISSN $2007-7467$

biorhythmic state is relevant because, mainly, it allows locating and relocating workers in periods exposed to risks and, secondly, avoiding the increase in the insurance of the occupational risk premium.

Keywords: accidents at work, biorhythms, circadian cycles, occupational hazards.

\section{Resumo}

As organizações industriais ou de transformação aspiram a contratar trabalhadores para realizar atividades nos processos de produção em condições ideais. No entanto, contextos diferentes podem alterar as condições e afetar seu status de biorritmo. Ao alterar esses fatores, favorece-se a ocorrência de acidente de trabalho. O objetivo deste trabalho é investigar a existência de uma relação entre ciclos circadianos com incidências de acidentes de trabalho nos sistemas de produção de entidades industriais ou de transformação. Neste estudo longitudinal, foram utilizadas as bases de dados fornecidas pela subdelegação do Instituto Mexicano del Seguro Social (IMSS) da zona leste do Estado do México nos anos de 2013, 2014 e 2015. estatística descritiva, a fim de saber o comportamento das variáveis da amostra. Os resultados sugerem que os ciclos circadianos compostos pelo estado físico, emocional e intelectual influenciam significativamente na ocorrência de acidentes de trabalho dentro da empresa. O exposto permite inferir que conhecer o estado biorrítmico é relevante porque, principalmente, permite localizar e recolocar trabalhadores em períodos de exposição a riscos e, em segundo lugar, evitar o aumento do seguro do prémio de risco do trabalho.

Palavras-chave: acidente de trabalho, biorritmos, ciclos circadianos, riscos ocupacionais. Fecha Recepción: Enero 2021 Fecha Aceptación: Julio 2021

\section{Introducción}

En el siglo XIX, los primeros estudios sobre los ciclos circadianos se realizaron en ciertos "ritmos" o "ciclos" de vida que luego fueron llamados biorritmos. La palabra biorritmo está compuesta por dos términos griegos, bios y rhythmos, que significan 'vida' y un constante o periódico 'ritmo'. La teoría del biorritmo define y mide tres fundamentales e importantes ciclos del género humano: el ciclo físico, el emocional y el intelectual.

Wilhelm Fliess, un respetado y conocido doctor de Berlín, comenzó a interesarse por los biorritmos en el año de 1890. Fliess, que había observado ciclos de 23 y 28 días en muchos de sus pacientes, comenzó a recoger estadísticas sobre las frecuencias periódicas de la fiebre, 


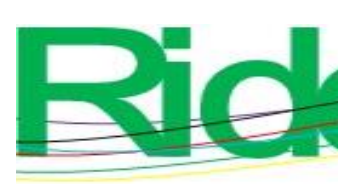

Revista Iberoamericana para la
Investigación y el Desarrollo Educativo ISSN $2007-7467$

\section{Marco teórico}

El Instituto Nacional de Ciencias Médicas Generales [Nigms, por sus siglas en inglés] (2017) define a los ritmos circadianos como cambios físicos, mentales y conductuales que siguen un ciclo diario, y que responden, principalmente, a la luz y a la oscuridad del ambiente de un organismo. Dormir por la noche y estar despierto durante el día es un ejemplo de un ritmo circadiano relacionado con la luz. Los ritmos circadianos se encuentran en la mayoría de los seres vivos, incluidos los animales, las plantas y muchos microbios diminutos. El estudio de los ritmos circadianos se llama cronobiología.

Arreaza y Arreaza (2001) especifican lo siguiente:

En todos los organismos vivos, desde las bacterias hasta los humanos, opera un reloj biológico sincronizado a 24 horas/día. Este reloj interior controla una gran variedad de funciones bioquímicas que se llaman fluctuaciones de ciclos, incluidos el del comportamiento, el del sueño y la actividad, que colectivamente se les llaman los ritmos circadianos (del latín: $\operatorname{circadia}=$ alrededor del día) (p. 31).

Por su parte, Silva (1984) señala que la historia de los biorritmos se remonta al año 1729, cuando Jean Jacques d'Ortous de Mairan observó que una planta llamada mimosa púdica abría sus hojas durante el día y las cerraba durante la noche. Parecía claro que aquella planta "reaccionaba a la luz" con algún tipo de movimiento reflejo, pero a Mairan se le ocurrió encerrar a aquella planta en un armario oscuro y pudo comprobar que la misma continuaba abriendo y cerrando sus hojas sin ningún tipo de estímulo lumínico. A partir de ahí se abrió la posibilidad de suponer que los seres vivos podríamos poseer alguna especie de "relojes biológicos" endógenos.

Fliess (1906) observaba que los niños podían estar expuestos a las mismas enfermedades y, sin embargo, permanecer inmunes durante días enteros. Luego de considerar el inicio de la enfermedad y su evolución, llegó a la conclusión que no todos los enfermos presentaban similares cuadros al mismo tiempo. Así, funda su teoría sobre los ritmos biológicos. Fliess (1906) comprobó que el ritmo físico (masculino) duraba 23 días y el ritmo emocional (femenino) 28 días, que ambos estaban presentes en cada célula del organismo humano y que tenían relación con la fecha de nacimiento, marcando altas y bajas. Sigmund Freud (1986) creyó en la teoría de Fliess, sostuvo que sería una apertura en materia biológica y le hizo llegar informaciones sobre los ciclos de su propia vida y la de su familia, a partir de 


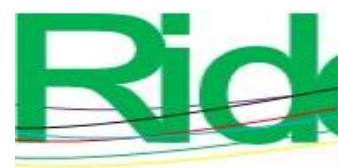

Revista Iberoamericana para la
Investigación y el Desarrollo Educativo
ISSN $2007-7467$

los cuales había comprobado alteraciones de salud que fluctuaban según los ciclos. Swoboda (1897, citado en Tomen, 2001) retomó la revolucionaria teoría, profundizó las investigaciones, las defendió y escribió acerca de su aplicación. Swoboda confirmó la existencia de los citados ciclos y le dio importancia a los días críticos que se originan cuando el ritmo de alto a bajo o viceversa.

Winstead, Schwartz y Bertrand (1981) apuntan que fue Alfred Teltscher quien añadió un tercer ciclo de 33 días a partir de haber detectado que la capacidad intelectual de sus alumnos (comprensión, creatividad, concentración, etc.) variaba clínicamente durante este periodo. Desafortunadamente, no dejó materiales publicados. Años más tarde comienza a utilizarse el biorritmo en Alemania, Japón, Estados Unidos, Canadá, Francia y Latinoamérica. Ahora, gracias a los avances tecnológicos, es posible conocer el estado del caudal energético en el orden físico, emocional y mental en cualquier momento, saber cómo canalizar las energías, comprender por qué se originan ciertas situaciones, respetando nuestro reloj biológico. Además, es posible sabes que la duración de los tres ritmos circadianos es la siguiente:

- $\quad$ Ritmo físico = 23 días, 11 1 1/2 días positivos, 11 1⁄2 días en baja.

- $\quad$ Ritmo emocional = 28 días, 14 días positivos, 14 días en baja.

- $\quad$ Ritmo intelectual = 33 días, 16 1⁄2 días positivos, 16 1⁄2 días en baja.

El biorritmo se refiere a tres ciclos biológicos activos en todos los seres humanos: físico, emocional e intelectual. Estos ciclos determinan en las personas ciertos estados favorables o desfavorables que pueden ser previstos y así poder planificar y orientar de manera más conveniente y eficaz las actividades en todos los aspectos de la vida.

Cada uno de los ciclos biológicos es calculado mediante ecuaciones de curvas sinusoidales, donde $t$ indica el número de días transcurridos desde la fecha de nacimiento de la persona (ver figura 1). 


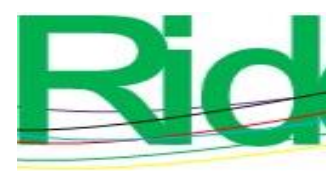

Revista Iberoamericana para la Investigación y el Desarrollo Educativo ISSN $2007-7467$

Figura 1. Curva sinusoidal

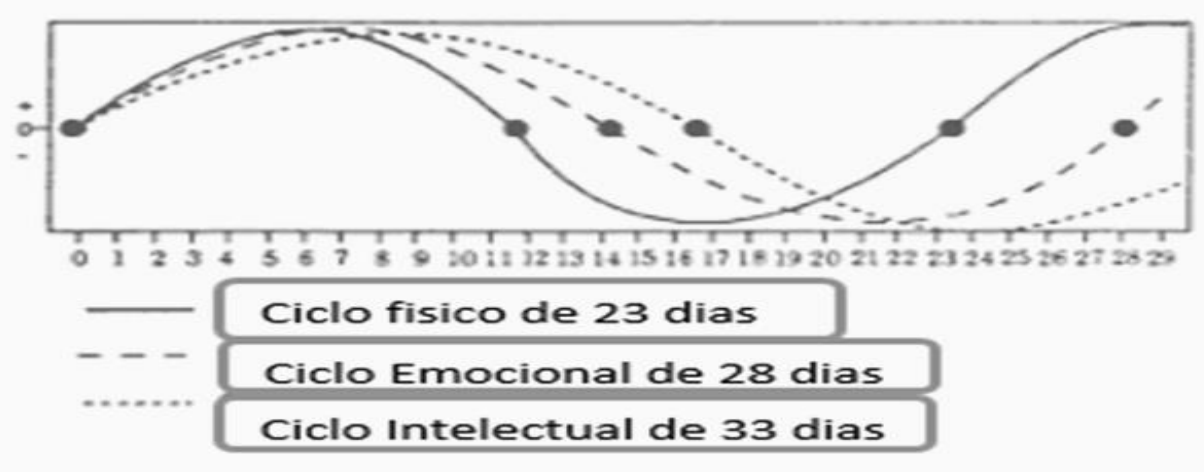

Fuente: Cardinali (1994)

- $\quad$ Ciclo físico: $\operatorname{sen}(2 \pi \mathrm{t} / 23)$,

- $\quad$ Ciclo emocional: $\operatorname{sen}(2 \pi t / 28)$

- $\quad$ Ciclo intelectual: sen $(2 \pi \mathrm{t} / 33)$,

Los investigadores Swoboda, Fliess y Teltscher, propulsores de la teoría del biorritmo, indican que mediante esas ecuaciones es posible conocer el estado energético tanto en el orden físico como emocional e intelectual de cualquier individuo en una fecha determinada.

Feldman (1995) define a los ritmos circadianos como procesos biológicos que se repiten en ciclos de aproximadamente 24 horas. El sueño y la vigilia, por ejemplo, ocurren naturalmente al ritmo de marcapasos internos. El ritmo circadiano se observa principalmente en la alternancia entre sueño y vigilia, pero también es posible observarlo en otras variables biológicas, como la temperatura corporal, la secreción de hormonas, las funciones cognitivas e incluso las emociones (Silva, 2010). Otras diversas funciones corporales, como la de la temperatura del cuerpo, funcionan asimismo con base en ritmos circadianos. Los ritmos circadianos son estados muy fuertes.

Teniendo en cuenta todo lo anterior, consideramos que el ciclo circadiano es un elemento importante sobre el que se debe reflexionar para poder prevenir un accidente de trabajo, debido a que cada ciclo del biorritmo está compuesto por dos semiciclos, uno positivo y otro negativo, ambos de la misma duración. Los ciclos se encuentran divididos en dos sectores: una parte positiva que inicia en cero e incrementa su valor positivo hasta alcanzar un pico máximo de uno, y a partir de allí desciende nuevamente hasta cero, donde tiene cabida el punto crítico de cambio y donde inicia el segundo sector, contrario al anterior, un descenso que parte de cero hasta alcanzar un valor de menos uno, su punto máximo, y 


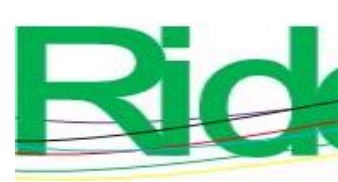

Revista Iberoamericana para la
Investigación y el Desarrollo Educativo
IsSN $2007-7467$

nuevamente va creciendo hasta alcanzar un valor de cero, y así se cumple un periodo completo de este ciclo.

Aun y cuando este concepto de ciclos circadianos tuvo su gran impacto en los 80 , con el paso del tiempo se fue olvidando e, infortunadamente, terminó mezclado entre situaciones esotéricas y magias populares, por lo que se perdió mucha seriedad en su tratamiento y aplicación. Sin embargo, de acuerdo con Rutter, Martin y McKnight (2002), el estudio de los ciclos y ritmos circadianos ha tomado suma importancia en las áreas de la conducta humana, por ejemplo, a partir del estudio del mecanismo del "aparato biorrítmico" y cómo regula el estado fisiológico del individuo acorde a su medio circundante. A este aparato biorrítmico se le estudia actualmente, incluso, a nivel molecular. De hecho, algunos autores lo definen como el aparato oscilatorio circadiano, el cual se busca modelar integrando información bioquímica, celular y psicológica.

Respecto al planteamiento anterior, se puede revisar el trabajo de Cermakian y Boivin (2003), quienes afirman que un gran número de variables psicosociales presentan ritmos circadianos o de 24 horas. Afirman, asimismo, que actualmente se han identificado genes dedicados a la regulación y generación de estos ritmos psicosociales y que algunas mutaciones en estos genes llevan a alteraciones del sueño y del estado de ánimo.

Por otra parte, Refinetti y Menaker (1992) han trabajado las relaciones entre el ritmo circadiano de la temperatura corporal (CRT) y definieron patrones oscilatorios del tipo ultradiano e infradiano de este CRT. Adicionalmente, estudiaron la relación entre este CRT y el ritmo circadiano del sistema de actividad locomotora, incluyendo su relación con el control nervioso.

Otro hallazgo importante relacionado con el estudio de estos biorritmos es el de Guo y Stein (2003), quienes, después de una revisión sistemática de 300 artículos científicos, encontraron conclusiones equivalentes en 52 de ellos. Así, Guo y Stein (2003) documentan una relación significativa entre los ritmos circadianos y el ritmo cardiaco y la presión arterial, así como con eventos patológicos psicosociales tales como isquemia miocárdica y muerte cardiaca repentina. Finalmente, recomiendan el estudio de los ritmos circadianos y su relación con el sistema cardiovascular como un medio de administración de la salud del paciente.

Una investigación más es la realizada por Hofstra y Weerd (2008). A sabiendas de que los ataques epilépticos tienen ciertos patrones, estudiaron la patología de la epilepsia y correlacionaron estos patrones con los ciclos circadianos. En su investigación encontraron 


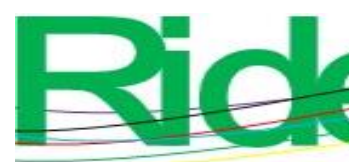

Revista Iberoamericana para la
Investigación y el Desarrollo Educativo
ISSN $2007-7467$

mecanismos para medir la fase del estado circadiano, tales como la luz, melatonina, temperatura corporal y el cortisol. En otras palabras, han logrado asincronizar-sincronizar el ritmo circadiano a través de estos marcadores biológicos. También buscaron correlacionar el estado biorrítmico del individuo al momento de un accidente, y una forma indirecta que encontraron es estimar el nivel del estado en cada uno de los elementos considerados, físico, emocional, intelectual. Pero, de acuerdo con estos investigadores, en realidad es el nivel de estos elementos bioquímicos (luz, melatonina, temperatura corporal y cortisol) lo que genera un estado de somnolencia, desatención, desconcentración, fatiga; en general, un estado psicoemocional de alto riesgo para tareas que involucran cualquier agente agresor (maquinaria, equipo, instrumentos).

Inclusive el estudio de estos ritmos circadianos se ha vinculado con el desarrollo de cáncer, a manera de ejemplo se puede ver las investigaciones de Savvidis y Koutsilieris (2012) y Zee, Attarian y Videnovic (2013).

Actualmente, hay una cantidad considerable y diversa sobre el estudio y aplicación de los ciclos circadianos, lo que nos permite concluir que:

- $\quad$ No son los biorritmos una cuestión esotérica sin fundamento científico.

- Son muy escasos los estudios que buscan correlacionar estos estados biorrítmicos con los accidentes industriales.

- Las aplicaciones disponibles actualmente solo indican el nivel del estado biorrítmico, no correlacionan estos indicadores con otras variables.

A partir de lo hasta aquí mencionado es posible comprender que un estudio correlacional extenso entre estos estados y el momento de un accidente industrial es más que necesario. Asimismo, la disponibilidad de una base de datos extensa permite sustentar un estudio exploratorio exhaustivo. Este es, entre otros, el principal objetivo de esta investigación.

Por otra parte, los ciclos circadianos son un factor que posibilita el cuidado y protección física del trabajador en el desempeño de sus labores. Además, contribuye en la prevención de los accidentes de trabajo y, por consiguiente, promueve el cumplimiento de las disposiciones especificadas en la Ley Federal de Trabajo mexicana.

Las disposiciones laborales especifican que los accidentes de trabajo son heridas que imposibilitan el bienestar del trabajador y que limitan el cumplimiento de sus actividades dentro de la organización. Las enfermedades son causas que provocan el malestar físico o 


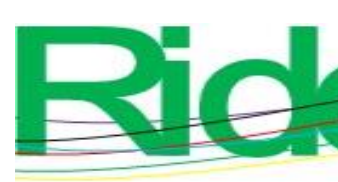

Revista Iberoamericana para la Investigación y el Desarrollo Educativo ISSN $2007-7467$

seguridad del trabajador. El objetivo del presente trabajo es investigar la relación entre los ciclos circadianos y los accidentes laborales en entidades industriales o de transformación.

\section{Descripción del método}

En este documento se reporta la primera fase de una investigación extensa que se está desarrollando sobre la relación/caracterización de los ciclos circadianos con accidentes en la industria, así como con los niveles de aprovechamiento del estudiantado de nivel superior.

Como una primera aproximación se buscó encontrar un patrón, a través de estadística descriptiva entre estos accidentes y el estado biorrítmico del trabajador, de manera que los resultados sirvan como base para un estudio más extenso, incluyendo análisis estadísticos más rigurosos.

Se solicitó, mediante oficio, a la subdelegación del IMSS de la zona oriente del Estado de México que proporcionara una base de datos. Esta base de datos recibida contiene información acerca de la fecha de nacimiento de los trabajadores, la fecha en que incurrieron los accidentes y el tipo de accidente de trabajo. Con esta información se realizó un análisis estadístico descriptivo sobre la ocurrencia de accidentes de trabajo y el estado de su ciclo circadiano.

Posteriormente, se elaboró el cálculo para determinar su ciclo de biorritmo en ese periodo y conocer si tenía relación directa con el accidente. Asimismo, se identificó el tipo de estado que presentaba vulnerabilidad. En este estudio se considera la vulnerabilidad como el punto en el que el ciclo cruza por cero, esto es, el ciclo positivo decrece antes de pasar a la fase negativa e igualmente crece antes de pasar a la fase positiva. El punto entre la fase negativa y positiva se considera como crítico, esto es, la teoría dice que en ese punto la persona es más vulnerable.

Con los datos de la fecha de nacimiento del trabajador y la fecha del accidente, se realizó un comparativo, considerando la ocurrencia como "exitosa" cuando la fecha del accidente se encuentra a +/-1 día del punto crítico (cero), en cualquiera de los tres biorritmos, físico, intelectual y emocional.

Los resultados finales muestran las proporciones de coincidencia de estas ocurrencias consideradas como exitosas para fines de este estudio. 


\section{Resultados}

Al revisar los datos estadísticos sobre los riesgos de trabajo ocasionados por la relación laboral, podemos identificar que existe un alto índice de accidentes derivados del mal desempeño de sus actividades, ya sea por cuestiones físicas, emocionales o intelectuales, de aquí que existe la posibilidad de relacionarlos con los ciclos circadianos y de esta manera poder determinar cuándo es el mejor momento para que el trabajador desempeñe sus actividades y viceversa, ya que los datos estadísticos afirman el alto índice de riesgos ocasionados, como lo señala la tabla 2.

Tabla 2. Riesgos de trabajo terminados registrados en el IMSS por año de ocurrencia, según tipo de riesgo

\begin{tabular}{|l|l|l|l|l|l|}
\hline Periodo & Total & $\begin{array}{l}\text { Accidentes } \\
\text { de trabajo }\end{array}$ & $\begin{array}{l}\text { Accidentes } \\
\text { en trayecto }\end{array}$ & $\begin{array}{l}\text { Enfermedad } \\
\text { es de trabajo }\end{array}$ & $\begin{array}{l}\text { No } \\
\text { especificado }\end{array}$ \\
\hline 2011 & 539418 & 424624 & 111135 & 3659 & 0 \\
\hline 2012 & 549595 & 427811 & 117352 & 4432 & 0 \\
\hline 2013 & 537435 & 412523 & 119299 & 5613 & 0 \\
\hline 2014 & 482408 & 366942 & 109874 & 5592 & 0 \\
\hline
\end{tabular}

Fuente: Secretaría del Trabajo y Previsión Social, con información de la Coordinación de Salud en el Trabajo, IMSS

La figura 2 especifica las estadísticas del IMSS que muestran la clasificación de los riesgos de trabajo y el grado de incidencia de cada uno de ellos de los años 2014 al 2015, y se observa que el riesgo que ocurre con mayor frecuencia es el de accidentes de trabajo. 


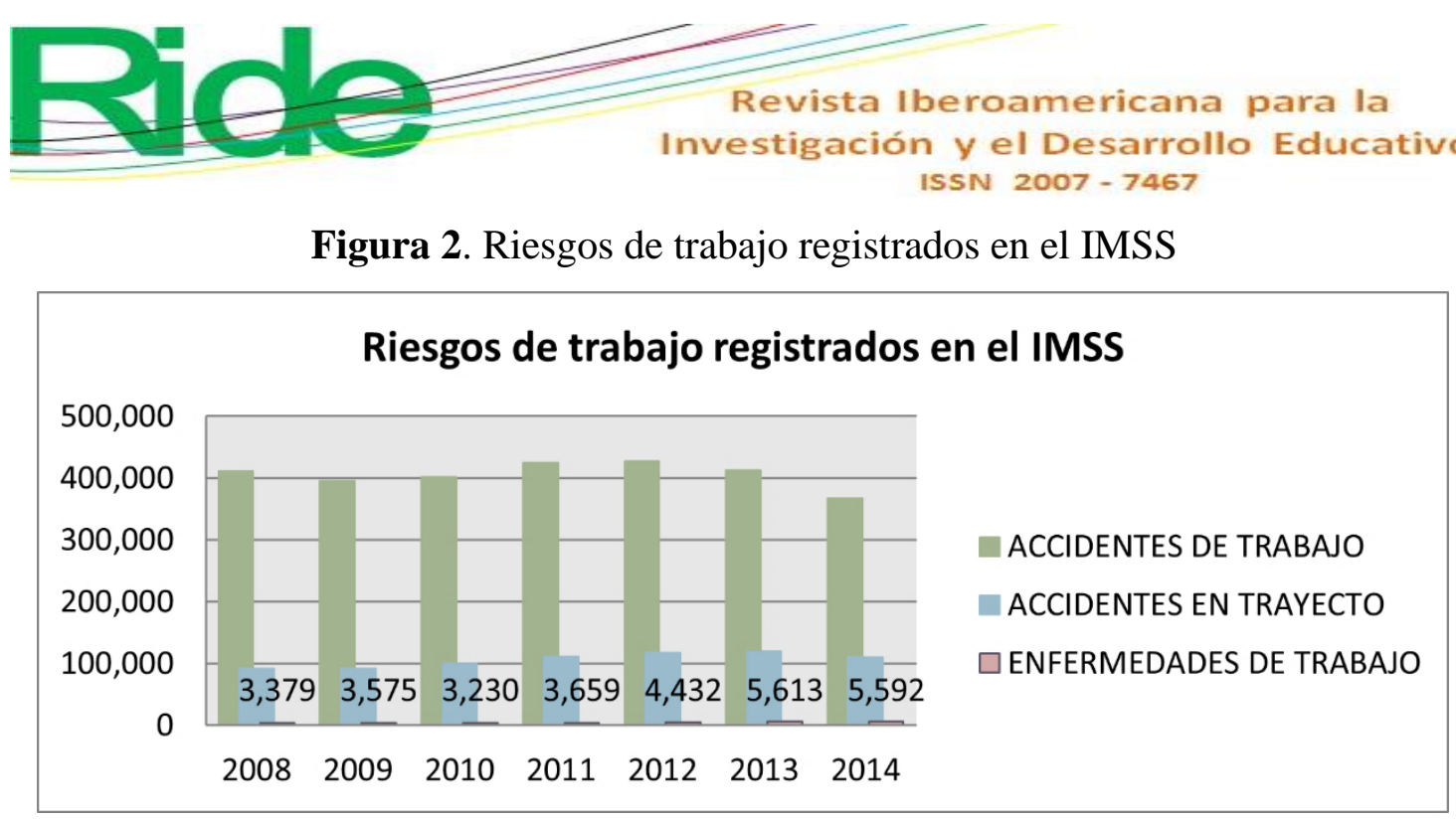

Fuente: Secretaría del Trabajo y Previsión Social con información de la Coordinación de Salud en el Trabajo, IMSS.

Los datos proporcionados por la delegación de Ecatepec de Morelos del IMSS sobre los trabajadores que han tenido accidentes de trabajo en los años 2013, 2014 y 2015 conforman $100 \%(n=34443)$ de los casos registrados, de los cuales $22 \%(n=7485)$ corresponden al año 2013, $49 \%(n=16826)$ al año 2014 y $29 \%(n=10132)$ de los casos fueron registrados en 2015 (tabla 3).

Tabla 3 Número de casos de accidentes de trabajo

\begin{tabular}{|c|c|c|}
\hline Años & Núm. de casos & $\%$ por escala \\
\hline 2013 & 7485 & $22 \%$ \\
\hline 2014 & 16826 & $49 \%$ \\
\hline 2015 & 10132 & $29 \%$ \\
\hline Total & 34443 & $100 \%$ \\
\hline
\end{tabular}

Fuente: Elaboración propia

Al realizar la depuración de trabajadores relacionados con la actividad industrial, se determinó que $11 \%(n=3820)$ de casos considerados para esta investigación ocurrieron en el año 2013, $12 \%(n=4238)$ fueron en el 2014 y $10 \%(n=3378)$ en el 2015 (tabla 4 ). Aquí observamos una variación en el incremento y disminución de los accidentes registrados durante los años de referencia, por lo cual no se puede predecir la tendencia de los accidentes de trabajo en los años subsecuentes. 


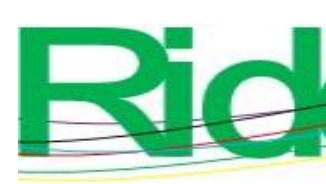

Revista Iberoamericana para la Investigación y el Desarrollo Educativo ISSN $2007-7467$

Tabla 4. Depuración de trabajadores relacionados con el área industrial

\begin{tabular}{|c|c|c|}
\hline Años & Núm. de casos & $\%$ por escala \\
\hline 2013 & 3820 & $11 \%$ \\
\hline 2014 & 4238 & $12 \%$ \\
\hline 2015 & 3378 & $33 \%$ \\
\hline Total & 11436 & $10 \%$ \\
\hline
\end{tabular}

Fuente: Elaboración propia

Respecto a los estados biorrítmicos emocional, físico e intelectual al momento del accidente, se encontró lo siguiente.

\section{Factor emocional}

Los biorritmos emocionales se refieren al estado de nuestras emociones, la vitalidad anímica, los sentimientos que varían según el estado de ánimo y el humor. En fase alta estamos más alegres; en fase baja, más apáticos; la fase crítica es la más propensa a las discusiones.

En este caso, los datos señalan que en el 2013, $77.51 \%(n=2961)$ representa al género masculino, y el punto crítico se encuentra en la fase negativa en el rango de -0.1 a -1 con un porcentaje acumulado de $37.67 \%(n=1439)$; mientras que para el año $2014,74.40 \%($ $n=3153$ ) del género masculino, y el punto crítico en un intervalo positivo de 0.1 a 1 , representando un porcentaje de $44.88 \%(n=1902)$, y para el año 2015, $76.58 \%(n=2587)$ conformado por el género masculino, y un punto crítico negativo del $37.03 \%(n=1251)$, comprendiendo, al igual que en el 2013, del -0.1 a -1 (tabla 5).

Respecto a los trabajadores del género femenino para el año 2013, representa el $22.49 \%(n=859)$, del cual el punto crítico es positivo, debido a que $10.79 \%(n=412)$ en un rango de 0.1 a 1 , para el $201425.60 \%(n=1,085)$ de mujeres el punto crítico se encuentra en un intervalo de $-0.1 \mathrm{a}-1$, representado por $14.70 \%(n=623)$, y para 2015, con un total de mujeres del $23.42 \%(n=791)$, el punto crítico es negativo con $11.34 \%(n=383)$ en un rango de -0.1 a -1 (tabla 5) 


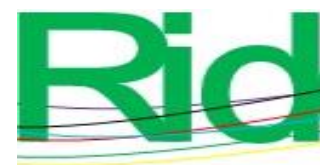

Revista Iberoamericana para la Investigación y el Desarrollo Educativo ISSN 2007 - 7467

Tabla 5. Factor emocional

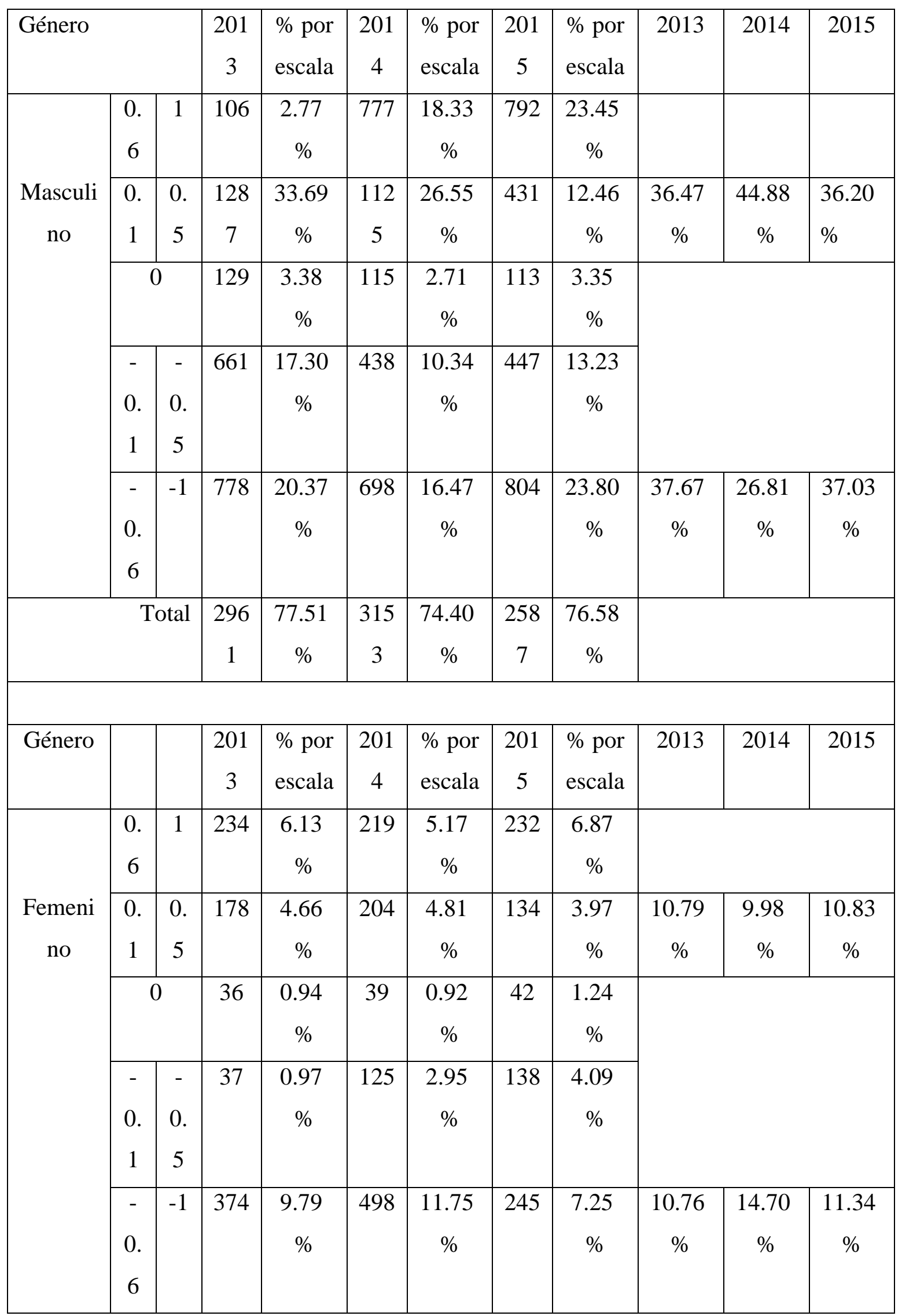




\begin{tabular}{|l|l|c|c|c|c|c|l|}
\hline & Total & 859 & 22.49 & 108 & 25.60 & 791 & 23.42 \\
$\%$ & 5 & $\%$ & & $\%$ & \\
\hline Total & 382 & $100 \%$ & 423 & $100 \%$ & 337 & $100 \%$ & \\
& 0 & & 8 & & 8 & & \\
\hline
\end{tabular}

Fuente: Elaboración Propia

\section{Factor físico}

El factor físico es un elemento que las empresas deben de considerar importante dentro de las áreas de trabajo, ya que se pueden ver afectados seriamente los trabajadores en las áreas de operación.

Los datos obtenidos muestran que los trabajadores que tuvieron un accidente de trabajo respecto del factor físico en 2013, considerando que de $100 \%(n=3820)$ de trabajadores, $77.51 \%(n=2961)$ pertenece al género masculino, obteniendo una suma acumulada positiva de $33.53 \%(n=1281)$ y estableciendo el punto crítico que va en un rango de 0.1 a 1 como porcentaje mayor, con $26.07 \%(n=996)$ en un rango negativo de -0.6 a -1 negativo.

De $100 \%(n=4238)$, para 2014, $74.40 \%(n=3153)$ pertenece al género masculino, identificando el punto crítico en un rango negativo del $-0.01 \mathrm{a}-1$, con $31.97 \%(n=1355)$ de suma acumulada y $23.24 \%(n=985)$ siendo el mayor porcentaje en un intervalo positivo de 0.6 a 1; mientras que para el año 2015, de $100 \%(n=3378)$ de trabajadores, $76.58 \%(n=$ 2587) pertenecen al género masculino, obteniendo un punto crítico negativo de $34.22 \%$ ( $n=$ 1156) de una suma acumulada en el rango de -0.01 a -1 , y $25.84 \%$ representando como mayor porcentaje al punto crítico en un rango positivo del 0.6 a 1 (tabla 6 ).

Considerando a los trabajadores del género femenino para el año 2013, representan $22.49 \%(n=859)$; obtuvieron un punto crítico acumulado positivo de $10.10 \%(n=336)$ en un rango de 0.1 a 1 y como porcentaje más alto del punto crítico $7.41 \%(n=283)$ con un intervalo de -0.6 a -1 negativo; para el $2014,25.60 \%(n=1085)$ de mujeres el punto crítico se encuentra en un intervalo acumulado de -0.1 a -1 , representado por $9.89 \%(n=409)$, y con $8.45 \%(n=358)$, representando el punto más alto en un rango de 0.6 a 1 positivo, y para 2015 , con un total de mujeres de $23.42 \%$ ( $n=791$ ), con un punto crítico positivo del $10.54 \%$ $(n=356)$ en un rango de 0.1 a 1 y el más alto con $7.55 \%(n=255)$ en un intervalo de 0.6 a 1 positivo (tabla 6 ). 


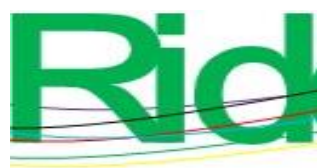

Es posible identificar que en los años de estudio el punto crítico ha sido en un rango positivo y negativo, por lo que se interpreta que el accidente de trabajo puede incurrir en cualquier intervalo, y ocasionar daños progresivos en la salud de los trabajadores e indemnizaciones mayores para las empresas.

Tabla 6. Factor físico

\begin{tabular}{|c|c|c|c|c|c|c|c|c|c|c|c|}
\hline Género & & & $\begin{array}{c}201 \\
3\end{array}$ & $\begin{array}{l}\% \text { por } \\
\text { escala }\end{array}$ & $\begin{array}{c}201 \\
4\end{array}$ & $\begin{array}{l}\% \text { por } \\
\text { escala }\end{array}$ & $\begin{array}{c}201 \\
5\end{array}$ & $\begin{array}{l}\% \text { por } \\
\text { escala }\end{array}$ & 2013 & 2014 & 2015 \\
\hline \multirow{5}{*}{$\begin{array}{c}\text { Masculi } \\
\text { no }\end{array}$} & \begin{tabular}{c|}
0. \\
6
\end{tabular} & 1 & 901 & $\begin{array}{c}23.59 \\
\%\end{array}$ & 985 & $\begin{array}{c}23.24 \\
\%\end{array}$ & 873 & $\begin{array}{c}25.84 \\
\%\end{array}$ & & & \\
\hline & \begin{tabular}{c|}
0. \\
1
\end{tabular} & $\begin{array}{l}0 . \\
5\end{array}$ & 380 & $\begin{array}{c}9.95 \\
\%\end{array}$ & 424 & $\begin{array}{c}10.00 \\
\%\end{array}$ & 363 & $\begin{array}{c}10.75 \\
\%\end{array}$ & $\begin{array}{c}33.53 \\
\%\end{array}$ & $\begin{array}{c}33.25 \\
\%\end{array}$ & $\begin{array}{l}36.59 \\
\%\end{array}$ \\
\hline & \multicolumn{2}{|c|}{0} & 202 & $\begin{array}{c}5.29 \\
\%\end{array}$ & 389 & $\begin{array}{c}9.18 \\
\%\end{array}$ & 195 & $\begin{array}{c}5.77 \\
\%\end{array}$ & & & \\
\hline & $\begin{array}{c}- \\
0 . \\
1\end{array}$ & $\begin{array}{l}- \\
0 . \\
5\end{array}$ & 482 & $\begin{array}{c}12.62 \\
\%\end{array}$ & 460 & $\begin{array}{c}18.85 \\
\%\end{array}$ & 342 & $\begin{array}{c}10.12 \\
\%\end{array}$ & & & \\
\hline & $\begin{array}{c}- \\
0 . \\
6\end{array}$ & -1 & 996 & $\begin{array}{c}26.07 \\
\%\end{array}$ & 895 & $\begin{array}{c}21.12 \\
\%\end{array}$ & 814 & $\begin{array}{c}24.1 \\
\%\end{array}$ & $\begin{array}{c}38.69 \\
\%\end{array}$ & $\begin{array}{c}31.97 \\
\%\end{array}$ & $\begin{array}{c}34.22 \\
\%\end{array}$ \\
\hline \multicolumn{3}{|c|}{ Total } & $\begin{array}{c}296 \\
1\end{array}$ & $\begin{array}{c}77.51 \\
\%\end{array}$ & $\begin{array}{c}315 \\
3\end{array}$ & $\begin{array}{c}74.40 \\
\%\end{array}$ & $\begin{array}{c}258 \\
7\end{array}$ & $\begin{array}{c}76.58 \\
\%\end{array}$ & & & \\
\hline Género & & & $\begin{array}{c}201 \\
3\end{array}$ & $\begin{array}{l}\% \text { por } \\
\text { escala }\end{array}$ & $\begin{array}{c}201 \\
4\end{array}$ & $\begin{array}{l}\% \text { por } \\
\text { escala }\end{array}$ & $\begin{array}{c}201 \\
5\end{array}$ & $\begin{array}{l}\% \text { por } \\
\text { escala }\end{array}$ & 2013 & 2014 & 2015 \\
\hline \multirow{3}{*}{$\begin{array}{c}\text { Femeni } \\
\text { no }\end{array}$} & \begin{tabular}{c|}
0. \\
6
\end{tabular} & 1 & 244 & $\begin{array}{c}6.39 \\
\%\end{array}$ & 358 & $\begin{array}{c}8.45 \\
\%\end{array}$ & 255 & $\begin{array}{c}7.55 \\
\%\end{array}$ & & & \\
\hline & \begin{tabular}{l|}
0. \\
1
\end{tabular} & $\begin{array}{l}0 . \\
5\end{array}$ & 142 & $\begin{array}{c}3.72 \\
\%\end{array}$ & 210 & $\begin{array}{c}4.96 \\
\%\end{array}$ & 101 & $\begin{array}{c}2.99 \\
\%\end{array}$ & $\begin{array}{c}10.10 \\
\%\end{array}$ & $\begin{array}{c}13.40 \\
\%\end{array}$ & $\begin{array}{c}10.54 \\
\%\end{array}$ \\
\hline & \multicolumn{2}{|c|}{0} & 54 & $\begin{array}{c}1.41 \\
\%\end{array}$ & 98 & $\begin{array}{c}2.31 \\
\%\end{array}$ & 61 & $\begin{array}{c}1.81 \\
\%\end{array}$ & & & \\
\hline
\end{tabular}




\begin{tabular}{|c|c|c|c|c|c|c|c|c|c|c|c|}
\hline & & & & & & $\begin{array}{r}\text { Rev } \\
\text { nvesti }\end{array}$ & $\begin{array}{l}\text { sta I } \\
\text { ació }\end{array}$ & $\begin{array}{l}\text { eroan } \\
\text { y el } \\
\text { SSN } 20\end{array}$ & $\begin{array}{l}\text { ericar } \\
\text { esarrc } \\
-746\end{array}$ & $\begin{array}{l}\text { par } \\
\text { o Ec }\end{array}$ & $\begin{array}{l}\text { a } \\
\text { ativo }\end{array}$ \\
\hline & $\begin{array}{l}- \\
0 . \\
1\end{array}$ & $\begin{array}{l}- \\
0 . \\
5\end{array}$ & 136 & $\begin{array}{c}3.56 \\
\%\end{array}$ & 207 & $\begin{array}{c}4.88 \\
\%\end{array}$ & 120 & $\begin{array}{c}3.55 \\
\%\end{array}$ & & & \\
\hline & $\begin{array}{c}- \\
0 . \\
6\end{array}$ & -1 & 283 & $\begin{array}{c}7.41 \\
\%\end{array}$ & 212 & $\begin{array}{c}5.00 \\
\%\end{array}$ & 254 & $\begin{array}{c}7.52 \\
\%\end{array}$ & $\begin{array}{c}10.97 \\
\%\end{array}$ & $\begin{array}{c}9.89 \\
\%\end{array}$ & $\begin{array}{c}11.07 \\
\%\end{array}$ \\
\hline & & tal & 859 & $\begin{array}{c}22.49 \\
\%\end{array}$ & $\begin{array}{c}108 \\
5\end{array}$ & $\begin{array}{c}25.60 \\
\%\end{array}$ & 791 & $\begin{array}{c}23.42 \\
\%\end{array}$ & & & \\
\hline Total & & & $\begin{array}{l}382 \\
0\end{array}$ & $100 \%$ & $\begin{array}{c}423 \\
8\end{array}$ & $100 \%$ & $\begin{array}{c}337 \\
8\end{array}$ & $100 \%$ & & & \\
\hline
\end{tabular}

Fuente: Elaboración propia

\section{Factor intelectual}

Este factor influye en la memoria y capacidad de razonar. Cabe puntualizar que en su fase positiva de 16 días y medio aumenta la capacidad para los estudios y todo lo que requiera atención y concentración; a diferencia de lo que sucede durante la fase negativa, los siguientes 16 días y medio siguiente, donde hay una disminución cognoscitiva (cansancio mental).

Evaluando a los trabajadores que incurrieron en un accidente, los resultados revelan que en el año $201377.51 \%(n=2961)$ representa al género masculino, analizando que el punto crítico se encuentra en la fase negativa en el rango de -0.1 a -1 con un porcentaje acumulado de $40.08 \%(n=1531)$, colocándose en mayor porcentaje $25.86 \%(n=988)$ en un rango negativo de -0.6 a -1 . Para el año $2014,74.40 \%(n=3153)$ pertenecientes al género masculino, adquiriendo un punto crítico en un intervalo negativo de -0.1 a -1 , representando un porcentaje acumulado de $37.14 \%(n=1574)$ y $21.59 \%(n=915)$ el más alto en un intervalo de -0.6 a -1 negativo.

Para el año 2015, $76.58 \%(n=2,587)$ fue conformado por el género masculino, y obtuvo un punto crítico negativo acumulado de $37.74 \%(n=1275)$, con mayor porcentaje en un rango de -0.6 a -1 negativo de $23.33 \%(n=788)$ (tabla 7$)$.

Considerando a los trabajadores del género femenino que incurrieron en un accidente de trabajo para el año 2013, estos representan $22.49 \%(n=859)$, con un punto crítico positivo 


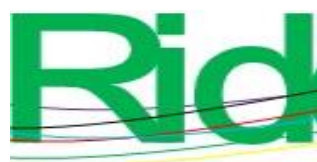

e

Revista Iberoamericana para la Investigación y el Desarrollo Educativo ISSN $2007-7467$

de $11.94 \%(n=456)$ acumulado en un rango de 0.1 a 1 y con $7.28 \%(n=278)$ el más alto en un rango de 0.6 a 1 positivo.

Para el 2014, $25.60 \%(n=1085)$ de mujeres registran el punto crítico en un intervalo de -0.1 a -1 , representado por $12.77 \%(n=541)$ acumulado y el más alto con $9.11 \%(n=$ 386) negativo en un rango de -0.6 a -1 negativo, y para 2015 , con un total de mujeres de $23.42 \%(n=791)$ el punto crítico es negativo, con $10.92 \%(n=369)$ acumulado en un rango de -0.1 a -1 , identificando el más alto con $7.02 \%(n=245)$ de un rango del 0.6 a 1 positivo (tabla 7).

Tabla 7. Factor intelectual

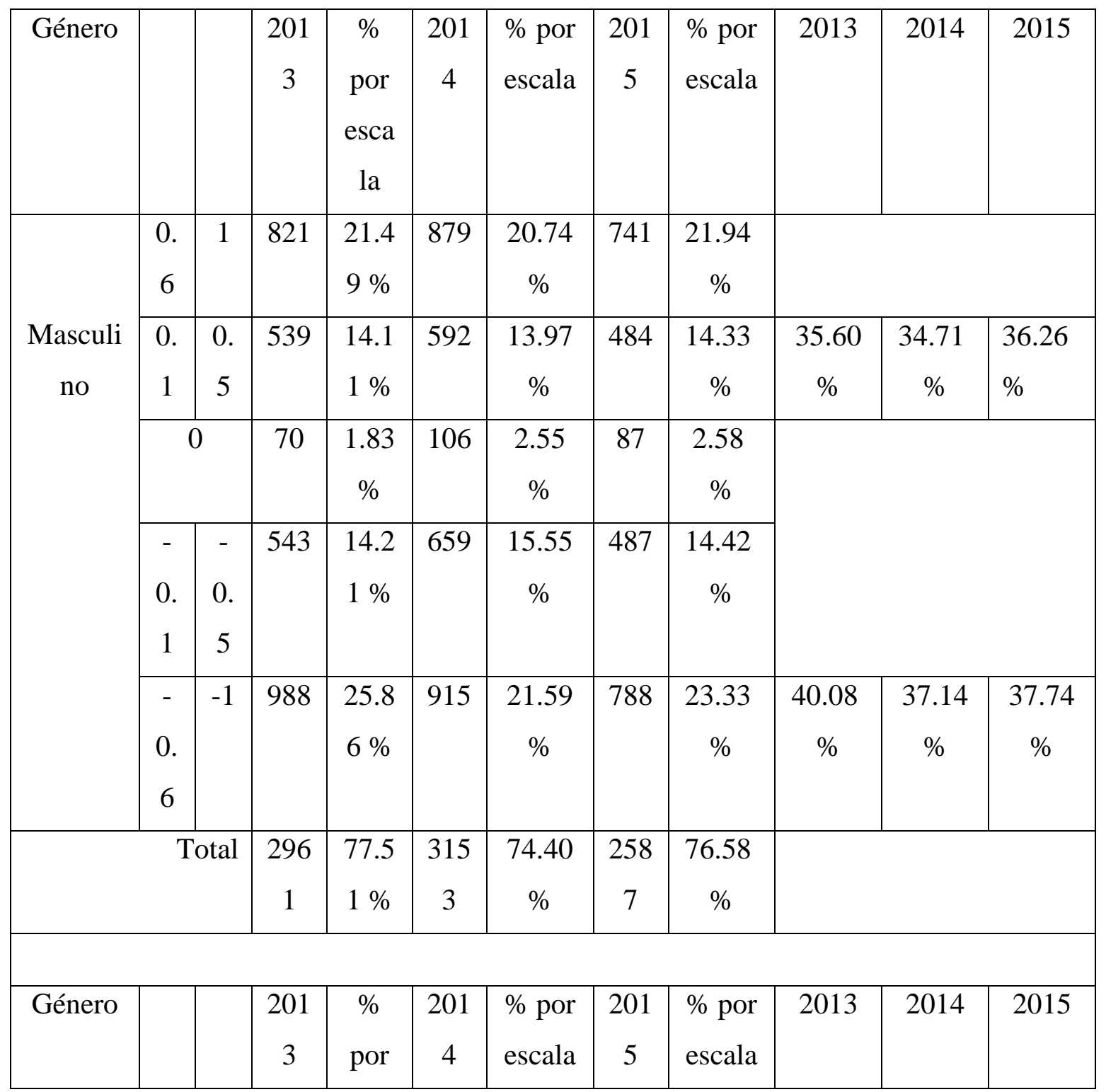




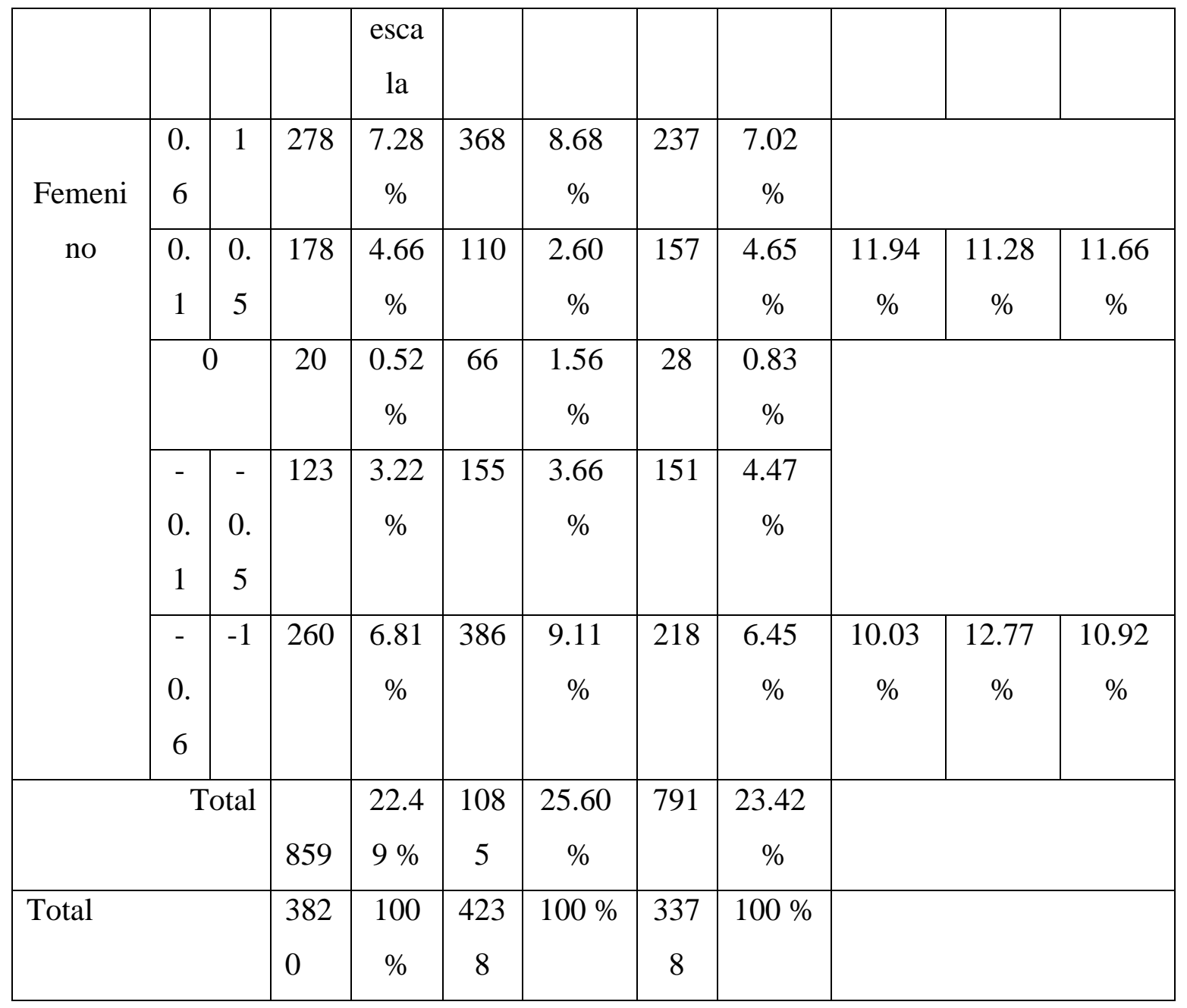

Fuente: Elaboración propia

\section{Discusión}

La teoría especifica que, para tener una inteligencia emocional estable, es necesario estar físicamente bien, ya que el estado de nuestras emociones proporciona vitalidad anímica. Los sentimientos varían según el estado de ánimo y el humor, estos elementos son indispensables para armonizar esas tendencias.

En el año 2013 un mayor número de trabajadores hombres sufrieron un accidente de trabajo encontrándose en la fase negativa. Respecto al género femenino, se ubica el mayor porcentaje de accidentes en la fase positiva. Para el 2014, la mayor relevancia está ubicada en la fase positiva en hombres y en la fase negativa para las mujeres. Y en 2015, para el género masculino y femenino se determinó que el mayor número de accidentes incurrieron en una fase negativa. En ningún caso el mayor número de trabajadores accidentados estaban en un punto crítico cero. Este análisis de datos del factor emocional se puede mencionar que 


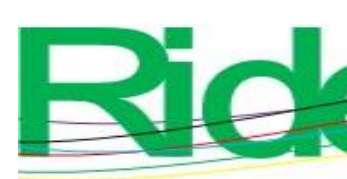

Revista Iberoamericana para la
Investigación y el Desarrollo Educativo
ISSN $2007-7467$

los accidentes no cumplen del todo con lo que especifica la teoría de que el punto crítico sea más propenso al momento de estar en fase negativa o al momento de estar en cero.

Respecto a los factores físicos, la teoría apunta que son factores ambientales que dependen de las propiedades físicas de los cuerpos. Los resultados estadísticos finales del análisis del factor físico muestran que el mayor número de accidentes para el año 2013 en el género masculino se ubica en la fase negativa, mientras que para el año 2014 y 2015 los accidentes se generaron dentro de la fase positiva. Lo anterior toma relevancia derivado de que en ese periodo se incumple con lo que especifica la teoría, debido a que en este caso los trabajadores sufren más accidentes en una fase positiva que en una negativa, en el caso de los hombres. Para el género femenino, en el año 2013 el mayor riesgo en los trabajadores que incurren en un accidente está ubicado en la fase negativa; en 2014 en la fase positiva, y en 2015 en la fase negativa. En ningún caso el mayor número de trabajadores accidentados estaban en un punto crítico cero, por lo que se considera que en este análisis de datos no necesariamente se cumple con la teoría de que el punto crítico es más propenso al momento de estar en fase negativa o al momento de estar en cero.

Por último, el factor intelectual nos especifica que es aquel que influye en nuestra memoria y capacidad de razonar. En relación con la base de datos, obtuvimos que en el año 2013 existió un mayor número de accidentes en el género masculino, que fue de $40.08 \%$, encontrándose en la parte negativa; para el género femenino, fue mayor la parte positiva, obteniendo un mayor porcentaje de $11.94 \%$, disminuyendo un poco los accidentes de trabajo a $10.03 \%$.

Para el año 2014, en ambos géneros se presentaron más accidentes debido a que se encontraron mayores trabajadores en su fase negativa: para el género masculino fue de $37.14 \%$ y el femenino de $12.77 \%$. Y en el año 2015, para el género masculino se encuentra en la fase negativa, con $37.74 \%$, y el femenino la mayor parte es para la positiva, $11.66 \%$, reduciendo en número de accidentes a $10.92 \%$.

Cabe destacar que las mujeres se encuentran más propensas a accidentes de trabajo en periodos negativos en dos de los factores, ya que de los tres años analizados se muestra como resultado que en dos tuvieron el accidente en periodo negativo en las fases emocionales y físicas, mientras que los hombres solo presentaron en un año afectaciones en el periodo negativo en estas dos fases y sus accidentes fueron recurrentes en periodos positivos. En comparación con el factor intelectual, las mujeres solo un año se encontraban en periodo 


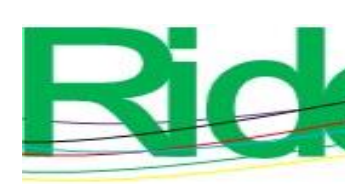

Revista Iberoamericana para la
Investigación y el Desarrollo Educativo
ISSN $2007-7467$

negativo y sus accidentes se presentaron en el periodo positivo, mientras que los hombres en esta fase todos sus accidentes se encontraron en periodo negativo.

\section{Conclusión}

Las organizaciones industriales o de transformación, al contar con trabajadores que se encuentran susceptibles a acontecimientos que limiten su rendimiento, buscan estrategias para disminuir riesgos. Una de las propuestas más viables ante este acontecimiento es la de la medición del ciclo de biorritmo de los trabajadores operativos y administrativos para medir el comportamiento de los ciclos físico, emocional e intelectual.

A partir de los resultados obtenidos en esta investigación, se observó que las mujeres son más susceptibles a presentar accidentes laborales en su ciclo físico y emocional negativo, mientras que los hombres son más susceptibles en periodos positivos. En cuanto al ciclo intelectual, los hombres son más susceptibles a accidentes en su periodo negativo, contrario a las mujeres, que se da en su ciclo positivo. Estos datos contrastan con lo que establece la teoría de que el punto crítico es cero o negativo. Como sea que fuere, se encontraron datos interesantes sobre cómo influyen los ciclos derivados de los géneros. La medición del ciclo biológico permite a las organizaciones conocer los periodos de posible riesgo dentro de la entidad con la finalidad de prevenir el riesgo. Es importante que los directivos de las empresas y profesionales de las áreas estudien estos factores para generar estrategias que mejoren el rendimiento del trabajador $\mathrm{y}$, por consiguiente, la productividad de la organización, disminuyendo las compensaciones de pago por accidentes.

Siguiendo con esta línea de investigación, se pretende investigar y analizar si hay relación directa entre el tipo de ciclo circadiano, el periodo de ocurrencia y el género, así como analizar las herramientas existentes que desarrollan el cálculo automatizado de los ciclos, proponer una aplicación que mida y evalúe los ciclos biológicos y conocer su usabilidad dentro de las empresas industriales, con el propósito de realizar una planeación estratégica como medida de prevención en la ocurrencia de los accidentes de trabajo. 


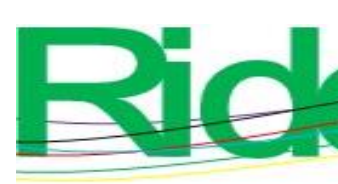

Revista Iberoamericana para la
Investigación y el Desarrollo Educativo
ISSN $2007-7467$

\section{Líneas de investigación propuestas}

Los resultados sugieren una correlación importante, sin embargo, es necesario considerar otras variables:

- $\quad$ El género. La estadística descriptiva sugiere una diferencia importante en las ocurrencias entre hombres y mujeres en los tres estados biorrítmicos estudiados.

- Debe incluirse una matriz de correlación, de manera que se estudie, matemáticamente, la significancia entre estas variables.

- Al parecer, la teoría del "punto crítico cero" no es suficiente. Es menester estudiar otras variables, tales como la combinación de los estados biorrítmicos, el sentido del estado al momento del accidente (creciente, decreciente, pendiente de la curva).

\section{Referencias}

Arreaza, R. y Arreaza, R. (2002). El ritmo circadiano: base molecular. Aspecto clínicos y laborales. Gaceta Médica de Caracas, 110(1), 31-34.

Cardinali, D. P., Jordá, J. y Sánchez, E. J. (1994). Introducción a la cronobiología: fisiología de los ritmos biológicos. España: Universidad de Cantabria.

Cermakian, N. and Boivin, D. (2003). A molecular perspective of human circadian rhythm disorders. Brain Research Reviews, 42(3) 204-220. Recuperado de https://doi.org/10.1016/S0165-0173(03)00171-1.

Feldman, R. (1995). Psicología con aplicaciones para Iberoamérica. México: McGraw-Hill.

Fliess, W. (1906). Der Ablauf des Lebens: Grundlegung zur exakten Biologie. Deutschland: Franz Deuticke.

Freud, S. (1986). Las cartas completas de Sigmund Freud a Wilhelm Fliess, 1887-1904. Buenos Aires, Argentina: Amorrortu editores.

Guo, Y. and Stein, P. K. (2003). Circadian rhythm in the cardiovascular system: chronocardiology. American Heart Journal, 145(5), 779-786. Recuperado de https://doi.org/10.1016/S0002-8703(02)94797-6.

Hofstra, W. and Weerd, A. (2008). How to assess circadian rhythm in humans: A review of literature. Epilepsy \& Behavior, 13, 438-444. Recuperado de https://doi.org/10.1016/j.yebeh.2008.06.002. 


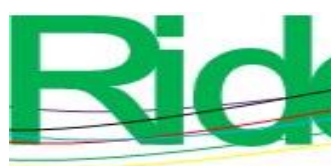

Revista Iberoamericana para la Investigación y el Desarrollo Educativo ISSN $2007-7467$

Instituto Nacional de Ciencias Médicas Generales [Nigms]. (2017). Hoja informativa sobre los ritmos circadianos. Recuperado de https://www.nigms.nih.gov/education/Documents/Spanish_circadian.pdf.

Presidencia de la República. (2019). Ley Federal de Trabajo. Diario Oficial de la Federación. Recuperado de http://www.diputados.gob.mx/LeyesBiblio/ref/lft.htm.

Refinetti, R. and Menaker, M. (1992). The circadian rhythm of body temperature. Physiology \& Behavior, 51(3), 613-637. Recuperado de https://doi.org/10.1016/00319384(92)90188-8.

Rutter, J., Martin, R. and McKnight, S. L. (2002). Metabolism and the Control of Circadian Rhythms. Annual Review of Biochemistry, 71, 307-331. Recuperado de https://www.annualreviews.org/doi/abs/10.1146/annurev.biochem.71.090501.14285 7.

Savvidis, C. and Koutsilieris, M. (2012). Circadian Rhythm Disruption in Cancer Biology. Molecular Medicine, 18, 1249-1260. Recuperado de https://10.2119/molmed.2012.00077.

Silva, L. (1984). Teoría de los biorritmos ¿resurgimiento de un mito? Revista Cubana de Administración de Salud, 10(4), 333-340.

Silva, F. (2010). Trastornos del ritmo circadiano del sueño: fisiopatología, clasificación y tratamientos. Revista Memoriza, (7), 1-13.

Tomen, G. S. (2001). Biorritmos-algo de historia. Recuperado de http://www.bibliotecapleyades.net/bioritmos/ciencia_biorritmos01.htm.

Winstead, D., Schwartz, W. and Bertrand E. (1981). Biorhythms: fact of superstition? American Journal of Psychiatry, 138(9), 1188-1192.

Zee, P., Attarian, H. and Videnovic, A. (2013). Circadian rhythm abnormalities. Continuum, 19(1), 132-147. 


\begin{tabular}{|c|c|}
\hline & $\begin{array}{l}\text { Revista Iberoamericana para la } \\
\text { Investigación y el Desarrollo Educativo } \\
\text { ISSN } 2007-7467\end{array}$ \\
\hline Rol de Contribución & Autor (es) \\
\hline Conceptualización & $\begin{array}{l}\text { Alfonso Aldape Alamillo (Principal); Juan Pedro Benítez } \\
\text { Guadarrama (Igual); Francisco Zorrilla Briones (Apoya) }\end{array}$ \\
\hline Metodología & $\begin{array}{l}\text { Juan Pedro Benítez Guadarrama (Principal); Lizette Alvarado } \\
\text { Tarango (Apoya) }\end{array}$ \\
\hline Software & $\begin{array}{l}\text { Juan Pedro Benítez Guadarrama (Principal); Manuel Alonso } \\
\text { Rodríguez Morachis (Igual) }\end{array}$ \\
\hline Validación & $\begin{array}{l}\text { Francisco Zorrilla Briones (Principal); Juan Pedro Benítez } \\
\text { Guadarrama (Igual) }\end{array}$ \\
\hline Análisis Formal & Juan Pedro Benítez Guadarrama \\
\hline Investigación & $\begin{array}{l}\text { Juan Pedro Benítez Guadarrama (Principal); Manuel Alonso } \\
\text { Rodríguez Morachis (Igual) }\end{array}$ \\
\hline Recursos & Lizette Alvarado Tarango \\
\hline Curación de datos & $\begin{array}{l}\text { Juan Pedro Benítez Guadarrama (Principal); Francisco Zorrilla } \\
\text { Briones (Igual) }\end{array}$ \\
\hline $\begin{array}{l}\text { Escritura - Preparación del } \\
\text { borrador original }\end{array}$ & $\begin{array}{l}\text { Juan Pedro Benítez Guadarrama (Principal); Alfonso Aldape } \\
\text { Alamillo (Apoya) }\end{array}$ \\
\hline $\begin{array}{l}\text { Escritura - Revisión y } \\
\text { edición }\end{array}$ & Francisco Zorrilla Briones \\
\hline Visualización & Juan Pedro Benítez Guadarrama \\
\hline Supervisión & $\begin{array}{l}\text { Francisco Zorrilla Briones (Principal); Juan Pedro Benítez } \\
\text { Guadarrama (Igual) }\end{array}$ \\
\hline Administración de Proyectos & Juan Pedro Benítez Guadarrama \\
\hline Adquisición de fondos & Lizette Alvarado Tarango \\
\hline
\end{tabular}

